\title{
Results of the De Vega Plasty in Tricuspid Insufficiencies Secondary to Mitral and Aortic Rheumatic Valve Diseases: Study of 58 Cases
}

\author{
Ciss Amadou Gabriel ${ }^{1, ~ *, ~ G a n d j i ~ W i l f r i d ~}{ }^{1}$, Diop Momar Sokhna ${ }^{1}$, Ba Papa Salmane ${ }^{1}$, \\ Leye Mohamed ${ }^{2}$, Diatta Souleymane ${ }^{1}$, Gaye Magaye ${ }^{1}$, Sene Etienne Birame ${ }^{3}$, Dieng Papa Adama ${ }^{1}$, \\ Ndiaye Assane', Ndiaye Mouhamadou ${ }^{1}$ \\ ${ }^{1}$ Service de Chirurgie Thoracique et Cardiovasculaire CHNU FANN, Department of Thoracic and Cardiovascular Surgery FANN University \\ Hospital, Dakar, Senegal \\ ${ }^{2}$ Service de Chirurgie Thoracique et Cardiovasculaire CHNUFANN, Department of Cardiology FANN University Hospital, Dakar, Senegal \\ ${ }^{3}$ Service de Chirurgie Thoracique et Cardiovasculaire CHNUFANN, Department of Anesthesiology FANN University Hospital, Dakar, \\ Senegal
}

\author{
Email address: \\ cissgaby@yahoo.fr (C. A. Gabriel), elowilly@yahoo.fr (G. Wilfried), momarsokhna08@yahoo.fr (D. M. Sokhna), \\ mansalb@yahoo.fr (Ba P. Salmane), leyemohamed@gmail.com (L. Mohamed), soul.diatta@yahoo.fr (D. Souleymane), \\ mgaye99@yahoo.fr (G. Magaye), biramsene@hotmail.com (S. E. Birame), padiengsala@yahoo.fr (D. P. Adama), \\ lazanat@yahoo.fr (N. Assane), mondiaye@orange.sn (N. Mouhamadou) \\ ${ }^{*}$ Corresponding author
}

\section{To cite this article:}

Ciss Amadou Gabriel, Gandji Wilfrid, Diop Momar Sokhna, Ba Papa Salmane, Leye Mohamed, Diatta Souleymane, Gaye Magaye, Sene Etienne Birame, Dieng Papa Adama, Ndiaye Assane, Ndiaye Mouhamadou. Results of the De Vega Plasty in Tricuspid Insufficiencies Secondary to Mitral and Aortic Rheumatic Valve Diseases: Study of 58 Cases. International Journal of Cardiovascular and Thoracic Surgery. Vol. 3, No. 2, 2017, pp. 5-8. doi: 10.11648/j.ijcts.20170302.11

Received: February 23, 2017; Accepted: March 13, 2017; Published: March 24, 2017

\begin{abstract}
Assess the short, medium and long-term efficiency of the De Vega tricuspid plasty. The study was retrospective (7 years and 11 months) and included all patients operated for valve diseases associated with functional tricuspid regurgitation (TR). The Epi Info software was used for descriptive analysis and logistic regression. There was a total of 58 patients with an average age of 26 years. In preoperative transthoracic echocardiography (TTE), 44.6\% of patients had stage III TR. After De Vega plasty, the immediate postoperative TTE showed no tricuspid leak in $23.5 \%$ of patients, a minimal leak in $62 \%$ of patients and a moderate leak in $14.5 \%$ of patients. After a 41 -month follow-up, $25 \%$ of living patients had no more TR and $30.56 \%$ had minimal insufficiency. Moderate insufficiency persisted in $30.56 \%$ of cases, and severe in $13.89 \%$ of cases. All patients with severe long-term TR had hemodynamic instability during reanimation $(\mathrm{p}=0.002)$. De Vega plasty is a low-cost technique that does not extend the surgical time. It is adapted to the functional tricuspid regurgitation surgery in disadvantaged areas.
\end{abstract}

Keywords: De Vega Plasty, Tricuspid Regurgitation, Cardiac Surgery

\section{Introduction}

Tricuspid insufficiency is the least common cardiac valvulopathy (1). It appears in the form of a functional tricuspid regurgitation, secondary to a mitral and / or aortic valve diseases. The etiology is dominated by the rheumatic disorder (2). De Vega plasty is a technique for reconstructing the tricuspid ring. It consists of making a double suture over the tricuspid ring to shrink it, while avoiding the conduction zone.

The aim of this study is to evaluate the short, medium, and long-term efficiency of the De Vega tricuspid plasty.

\section{Method}

The study is retrospective and descriptive. Data were collected from records of patients operated for cardiac valve diseases. It took place over a period of 8 years (January 2007 
to December 2015) with an average follow-up duration of 41 months for each patient. All patients operated for mitral, and / or aortic valve diseases and for functional tricuspid insufficiency (De Vega plasty) were included in the study. The statistical analysis used the Epi Info software; the Data Analysis tab which presents several functionalities was used for the tabulation of the descriptive statistics and the crossings between the variables. The data obtained were compared to those obtained by the SPSS and Stata software. The P-values of the different crossings were obtained through the Stata software. These values are compared with those of SPSS. The chi-square test with the Pearson coefficient was used for the determination of P-values.

\section{Results}

\subsection{Clinical Exam}

The total number of patients was 58 , with $63 \%$ of female, so a sex ratio of 0.58 . The average age was $26 \mathrm{y} / \mathrm{o}$ (7-63 y/o). On average, the patient's dyspnea was at stage III (NYHA).

A history of recurrent angina and rheumatic fever was present in $77 \%$ and $65 \%$ of patients, respectively. All patients were on diuretic therapy.

\subsection{Para Clinical Exam}

On the chest $\mathrm{x}$-ray, the average cardiothoracic ratio (CTR) was 0.67 (0.5-0.84).

The electrocardiogram showed complete arrhythmia by atrial fibrillation (AFIB) in $48 \%$ of patients, and $9 \%$ had a branch block.

In transthoracic echocardiography (TTE), the diameter of the right ventricle was greater than $28 \mathrm{~mm}$ in $24.5 \%$ of cases. The tricuspid annular plane systolic excursion (TAPSE) had an average value of $17.05 \mathrm{~mm}(5-30 \mathrm{~mm})$. The average systolic ejection fraction (EF) was 63\% (42-83\%). Valve lesions were listed in Table 1.

Table 1. Echographic aspects of preoperative valve lesions.

\begin{tabular}{lllllll}
\hline & $\begin{array}{l}\text { Mitral } \\
\text { regurgitation }\end{array}$ & $\begin{array}{l}\text { Mitral } \\
\text { stenosis }\end{array}$ & $\begin{array}{l}\text { Aortic } \\
\text { regurgitation }\end{array}$ & $\begin{array}{l}\text { anterior leaflet } \\
\text { prolapse }\end{array}$ & $\begin{array}{l}\text { posterior leaflet } \\
\text { restriction }\end{array}$ & $\begin{array}{l}\text { Fusion of } \\
\text { commissure }\end{array}$ \\
\hline Yes & $72,41 \%$ & $55,56 \%$ & $60,71 \%$ & $74,55 \%$ & $69,09 \%$ & $54,55 \%$ \\
No & $27,59 \%$ & $44,44 \%$ & $39,29 \%$ & $25,45 \%$ & $30,91 \%$ & $45,5 \%$ \\
\hline
\end{tabular}

All patients had functional tricuspid insufficiency (Figure 1).

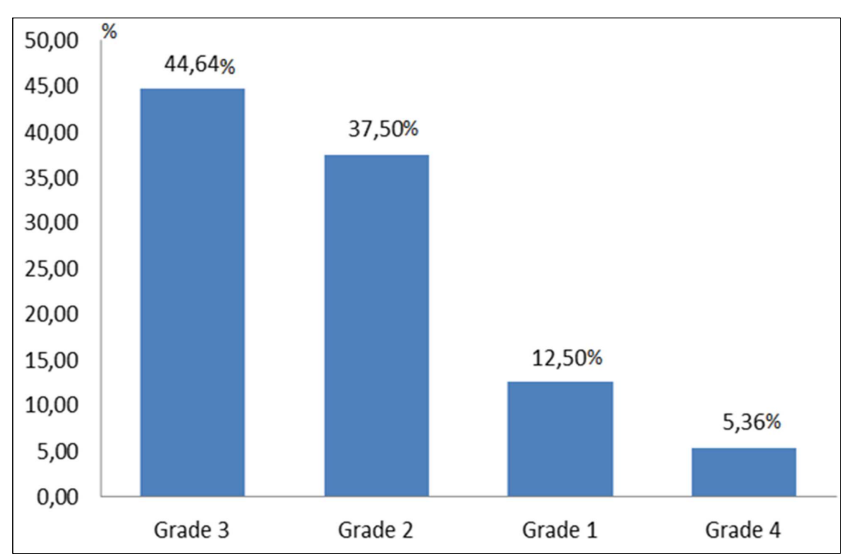

Figure 1. Distribution of preoperative tricuspid insufficiency.

The average tricuspid annular dimension (TAD) was $42.5 \mathrm{~mm}(28-63 \mathrm{~mm})$. The right ventricle (RV) - right atrium (RA) gradient was $55.7 \mathrm{~mm} \mathrm{Hg}$ on average. The average systolic pulmonary arterial pressure (PAPS) was $73 \mathrm{~mm} \mathrm{Hg}$ (28-110mm Hg).

\subsection{Surgery}

The approach was a vertical median sternotomy. The mitral valve was approached by a left atriotomy. The aortic valve was replaced by a transverse aortotomy, while the tricuspid valve was repaired from an incision in the right atrium. Mitral plasty was performed in $11 \%$ of patients and mitral valve replacement in $89 \%$ of patients. Compared to all valve replacements, mitral valve replacement was performed in $83 \%$ of patients, an aortic valve replacement in $2.38 \%$ of patients, and double valve replacement in $14.29 \%$ of the patients. A mechanical valve was implanted in $75.44 \%$ of the cases and the other patients received a bioprosthesis.

\subsection{Post Operative Period}

Immediate post-operative trans-thoracic echocardiography showed a lack of tricuspid leakage in $23.5 \%$ of patients, a minimal leak in $62 \%$ of patients and a moderate leak in $14.5 \%$ of patients. The systolic ejection fraction after extracorporeal circulation was an average of 50\% (25-66\%). The average mitral gradient was $4.10 \mathrm{mmHg}$ (1-13 $\mathrm{mmHg})$. Hemodynamically unstable patients were kept under amines in intensive care. Morbidity in intensive care was dominated by hemodynamic complications $(52 \%)$, pleuropulmonary complications (25\%) and cardiac rhythm disorders (18\%). Secondary complications were dominated by superficial surgical site infections $(6.25 \%)$, deep site infections $(8.33 \%)$ and pneumonia (4.17\%).

The postoperative mortality was $10.91 \%$ and the late mortality was $11.5 \%$. Causes of death were related to hemodynamic disorders, infectious complications, or rhythm disorders.

\subsection{Follow up}

After the De Vega tricuspid plasty, $89 \%$ of patients had stage I dyspnea. The percentage of patients without insufficiency after 3 months was $21 \%$. Minor insufficiency persisted in $63 \%$ of cases, moderate insufficiency in $10.8 \%$ of cases, and severe insufficiency in $5.26 \%$ of cases. The systolic pulmonary arterial pressure was $42 \mathrm{~mm} \mathrm{Hg}$ on average $(23-110 \mathrm{~mm} \mathrm{Hg})$. The follow-up lasted for around 41 
months (10-70 months). At the last cardiac ultrasound, $25 \%$ of the living patients had no insufficiency and $30.56 \%$ had minimal insufficiency. Moderate insufficiency persisted in $30.56 \%$ of cases, and severe in $13.89 \%$ of cases.

The results are summarized in Figure 2.

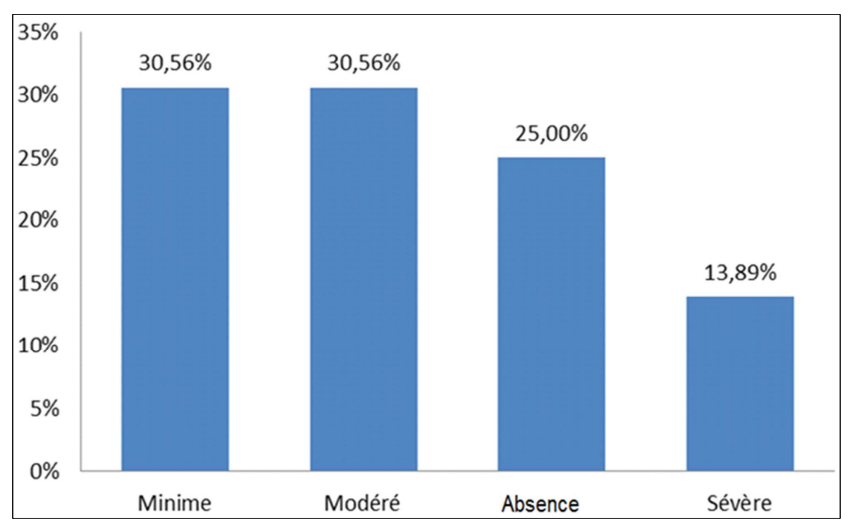

Figure 2. Patients distribution by long-term tricuspid insufficiency grade.

Of the 6 patients with severe long-term TI, only one patient had a grade 4 preoperative tricuspid leak, the remaining 5 had a grade 2 leak. Of the 18 patients who had moderate $\mathrm{TI}$, one $(5.6 \%)$ had a preoperative grade 4 tricuspid leak, five patients $(27.8 \%)$ had grade 3 leakage, and 8 patients $(44.4 \%)$ had a leak of grade 2 . A leak of grade 1 was found in 4 of them $(22.2 \%)$. Patients with a double valve or aortic valve alone did not have severe long-term TI, while $13.6 \%$ of patients with mitral valve replacement had severe leakage. All patients with severe long-term TI had hemodynamic instability on intensive care $(p=0.002)$. No deceased patients had grade 4 preoperative TI, but $75 \%$ had grade 3 TI $(p=0.058)$. Dobutamine $(p=0.151)$ was used in $14.71 \%$ of deaths cases and adrenaline $(p=0.001)$ in $45.45 \%$ of deaths.

\section{Discussion}

The De Vega tricuspid plasty is performed for functional tricuspid insufficiency in mitral and / or aortic valve surgery. Patients are rarely referred for isolated tricuspid valve surgery [3].

The rheumatic etiology is predominant in the underdeveloped countries and the population of patients is young, unlike the Western countries where degenerative causes are dominant [4].

Surgery improves the functional status of patients and thus their quality of life [5]. De Vega's annuloplasty was successful with a rate of $85.5 \%$ in the immediate postoperative period. In the literature, studies have demonstrated the effectiveness of De Vega's plasty in tricuspid insufficiencies due to functional dilation of the ring [6]. The results of Morishita's study [7] carried out on 408 patients indicated the non necessity of reoperation in $91.6 \%$ of patients operated by De Vega annuloplasty after a 15 -years follow-up. The improvement in hemodynamic factors related to the correction of left valvulopathies influenced the success of De Vega's plasty by favoring the reduction of the diameter of the tricuspid ring in the long term. In fact, the average pulmonary arterial pressure increased from $72,9 \mathrm{mmHg}$ preoperatively to $34.5 \mathrm{mmHg}$ in the long term, so a decrease of $38.4 \mathrm{mmHg}$. The grade of preoperative TI is a determining factor in the long-term effectiveness of De Vega's plasty. In the study by Ghodbane W. and al. [8], severe preoperative TI is also a predictor of failure of tricuspid plasties. All patients with severe preoperative TI had hemodynamic instability in intensive care.

The greater the correction of the left valvular lesions responsible for pulmonary arterial hypertension is complete, the greater the De Vega's plasty is long-term efficient [9]: long-term severe TI is not found in patients with double replacement Valvular disease while it was present in $13.8 \%$ of patients with mitral valve replacement. The severity of the preoperative dyspnea and the alteration of the left ventricular ejection fraction have a negative impact on the long-term results of De Vega's plasty [1]. In our study, the body mass index, the functional class of dyspnea, and atrial fibrillation do not influence postoperative mortality. However, these 3 elements may constitute factors of operative mortality. The severity of preoperative TI is a factor that influences postoperative mortality. Thus the correction of the annular dilatation before the appearance of more severe clinical or ultrasound TI has many benefits. Adrenaline administration is a factor of poor prognosis and influences post-operative mortality. Causes of death in patients who undertook tricuspid surgery are related to haemodynamic disorders and multi-visceral failure [10]. Some authors prefer the long-term superiority of prosthetic annuloplasty rather than De Vega's annuloplasty [11]. The implantation of a prosthetic ring improves the survival of the patients and reduces the risk of recurrence of a severe or moderate long-term TI compared to a classic or modified De Vega's plasty [12].

\section{Conclusion}

De Vega's plasty is a low-cost technique that does not extend the surgical time; it is suitable for surgery of functional tricuspid insufficiency in disadvantaged environments. However, prosthetic annuloplasty remains the most effective long-term technique for the correction of tricuspid insufficiencies.

\section{References}

[1] Kuwaki K. Morishita K. Tsukamoto M. Abe T. Tricuspid Valve Surgery for functional tricuspid valve regurgitation associated with left-sided valvular disease. European Journal of Cardio-Thoracic Surgery 2001; 20: 577-82.

[2] Yangni-Angate K. H., Ayegnon K. G., Meneas C. et al. Insuffisance tricuspidienne négligée au cours de la chirurgie valvulaire mitrale et/ou mitro-aortique. Ann. Afr. Chir. Thor. Cardiovasc. 2013; 8: 29-40. 
[3] Rogers J. H., Bolling S. P. The tricuspid Valve: current perspective an evolving management of tricuspid regurgitation. Circulation 2009; 119: 2718-25.

[4] Kangah M. K., Souaga K. A, Amani K. A. et al. Insuffisance mitrale rhumatismale de l'enfant: aspects anatomiques et chirurgicaux à propos de 84 cas. Ann. Afr. Chir. Thor. Cardiovasc 2009; 13: 11-14.

[5] Garg SK, Gosh PK, Misra B. Triple valve surgery in rheumatic heart disease cardiologie tropicale 1998, 24: 39-45.

[6] Morishita A, Kitamura $\mathrm{M}$ et al. Long-term results after De Vega's tricuspidannuloplasty. Jour Cardiovasc Surg 2002; 43: 773-7.

[7] Brugger J. J., Egloff L., Rothlin M., Kugelmeier J, Turina M, Senning. Tricuspid annuloplasty: results and complication. The Thorac Cardiovasc Surg 1982; 30: 284-87.

[8] Ghodbane W., Bey. M. Lejmi M. et al. La Chirurgie de l'insuffisance Tricuspide: quelles leçons tirer de notre enquête ? Tunisie Médicale 2012; 16: 200-6.
[9] Holper K., Hachnel J. C., Augustin N. et al. Surgery for tricuspid insufficiency: long-term follow up after De Vega annuloplasty. Thorac. Cardiovasc. Surg. 1993; 41: 1-8.

[10] Gariboldi V. Trousse. D et al. Chirurgie valvulaire après 75ans: évaluation desfacteurs de risque de mortalité hospitalière. Chir thorac card vasc. 2008; 12: 106-13.

[11] Khokisandi M., Banersee A., Singh H. et al. To a tricuspid annuloplasty ring significantly better than a De Vega's annuloplasty stitch when repairing severe tricuspid regurgitation. Interact Cardiovasc Thorac Surg 2012; 15: 129 35 .

[12] Tang G. H. L., David E. T., Singh S. K. et al. Tricuspid Valve Repair with an annuloplasty ring results in improves longterm out comes. Circulation 2006; 114: 1577-81. 